\title{
A Mathematical Model to Estimate the Position of Mobile Robot by Sensing Caster Wheel Motion
}

\author{
Jnana H. Amarendra ${ }^{1}$, Robins Mathew ${ }^{2}$ and Somashekhar S. Hiremath ${ }^{2 *}$ \\ ${ }^{1}$ Department of Electronics and Communication Engineering, Malnad College of Engineering, \\ Hassan-573201, India \\ ${ }^{2}$ Department of Mechanical Engineering, Indian Institute of Technology Madras, Chennai-600036, \\ India
}

\begin{abstract}
This paper describes the position estimation of mobile robot by sensing caster wheel motion. A mathematical model is developed to determine the position of mobile robot by sensing the angular velocity and heading angle of the caster wheel. Using the established equations, simulations were carried out using MATLAB version 8.6 to observe and verify the position coordinates of mobile robot and in turn obtain its trajectory. The simulation results show that the angular velocity of caster wheel and heading angle calculated from the sensor output readings with the help of inverse kinematics equations matches well with that of actual values given as input for simulation. Simulation result of tracking rectangular trajectory implies that the path traced by the mobile robot can also be determined from the sensor output readings. This concept can be implemented on a real mobile robot for estimation of its position.
\end{abstract}

Keywords: mobile robot, caster wheel, position estimation, kinematics, simulation.

\section{Introduction}

Creation of mobile robots has brought about prominent changes in various domains. In fact, with time mobile robots have substituted humans. They perform repetitive and dangerous tasks which humans prefer not to do or are unable to do because of inappropriate circumstances $[1,2]$. Evolution in the field of robotics has made tougher tasks much simpler with appreciable efficiency and precision [3]. Mobile robots are capable of navigating an uncontrolled environment without the requirement of physical or electromechanical guidance devices. They are a major focus of current research and find their importance in industrial, military, medical and security related areas $[4,5]$.

On considering their importance in real time applications, estimating their position can prove to be advantageous in many sectors. Valet parking without the aid of a valet is one

\footnotetext{
*Corresponding author: somashekhar@,iitm.ac.in
} 
such example. The car is transported to the desired position and orientation with the aid of mobile robots and laser range scanners [6,7]. With the current trend of automation in manufacturing technologies popularly known as Industry 4.0, autonomous mobile robots play a vital role. They provide flexible, efficient and integrated production by virtue of their locomotion feature [8]. In the search and rescue operation, mobile robots are operated to search victims on disaster scenes. These situations demand the implementation of teleoperated control and autonomous path following features on mobile robots for autonomous navigation [9]. Such autonomous mobile robots are not guided by physical path indicators and they are capable of dynamic route planning.

An appropriate hardware set up is required to sense the two dimensional motion of wheeled mobile robot to estimate its position. In this regard several techniques have been developed by many researchers. To mention a few, the Kalman Filter (KF) and the Extended Kalman Filter (EKF) was utilised for localisation of autonomous mobile robot based on Ackermann steering. It also states that the EKF is consistently better than the KF in estimating the robot position [10]. However, this technique lacks to represent ambiguities and in case of localisation failures, the ability to globally re-localize the robot is also absent. Another study involves installing the optical mouse sensors on mobile robots to detect its motion relative to the ground [11]. With the use of optical sensors, the position measurements are independent of kinematics of the mobile robot. Even though the optical sensors are cost effective and have high resolution sensing capability, they can be affected by errors. Placement and orientation of optical sensors on mobile robots must be accurate. In case of uneven surfaces, there are chances that the estimated position of robot is erroneous. In another research work, the Dynamic Markov Localization (DML) technique is applied to globally estimate the position of robot. This technique efficiently tracks its position and also recovers from localisation failures. The two approaches for localisation are local localisation and global localisation techniques. They can be distinguished based on the kind of localization problem they are designed to resolve. DML method combines the advantages of both the localisation approach for keeping track of the robot's position and global position estimation. This method uses probabilistic technique to achieve the efficiency and also determine when the robot lost track of its position [12]. Mobile robots can locate themselves and build three-dimensional model of their surrounding using scaleinvariant visual landmarks. Simultaneous Localization And Map Building (SLAMB) is an important factor for successful navigation of mobile robots in this regard. Mobile robots can locate themselves without prior knowledge of their initial position with the help of vision based SLAMB algorithm [13]. The mobile robots serve their purpose only when they are capable enough to safely navigate in populated and dynamic environments. Extensions of Markov localization algorithm can be employed in position estimation of mobile robots in dynamic environmental conditions [14]. This simple mechanism can be applied so that they can operate autonomously in densely populated environments.

In the current work, a new approach is carried out for estimating the position of mobile robots by sensing caster wheel motion. Caster wheels unlike standard wheels are usually a part of mobile robots which are not driven. They are freely turning wheels having two degree of freedom and can rotate. Generally, these wheels are used for balancing the mobile robots. Since these wheels are already a built in part of wheeled mobile robots, this approach requires less hardware installation on mobile robots. The concept behind the mechanism of a mechanical computer mouse can be considered in this context. It is observed that with proper hardware arrangement, this concept can be applied to estimate the position of mobile robot by sensing the castor wheel motion. A mechanical computer mouse consists of a track ball with two freely rotating rollers, located $90^{\circ}$ apart. The rotating rollers help in detection of forward-backward motion and left-right motion of the mouse. Likewise, the caster wheel is kept in contact with two perpendicularly arranged 
identical shafts connected to two sensors to sense caster wheel motion. Any translational motion causes movement in caster wheels which in turn can be used to estimate mobile robot's position. In this approach, slipping which is one of the common sources of measurement errors is taken care of.

With this hardware set up, an attempt can be made for estimating position of mobile robots by sensing motion of caster wheel. This technique provides a cost effective, simple hardware set up around caster wheels to estimate position and orientation of mobile robots. The results presented in this paper are of the pilot simulation study conducted to verify the concept for position estimation.

The current paper is organised as follows, the theoretical model for position estimation is deduced based on the concept of Kinematics and inverse Kinematics in Section II. Simulation results and discussions are presented in Section III followed by conclusions in Section IV.

\section{Theoretical modelling}

Consider caster wheel arrangement as shown in Fig. 1. Shafts of two sensors are assembled with caster wheel such that the two sensors connected to them are able to sense caster wheel motion in $\mathrm{X}$ and $\mathrm{Y}$ direction. The distance covered by caster wheel in one complete rotation, $s=r \theta$, where $r$ is the radius of caster wheel.

Since $\theta=\omega t$,

$$
s=r \omega t
$$

Here $t$ is the duration of rotation. Assuming that both the shafts connected to the sensors are identical, the distance covered by shafts in one complete rotation, $s_{1}$ is equal to the circumference of shafts,

Here $r_{l}$ is the radius of shafts,

$$
s_{1}=r_{1} \omega t
$$

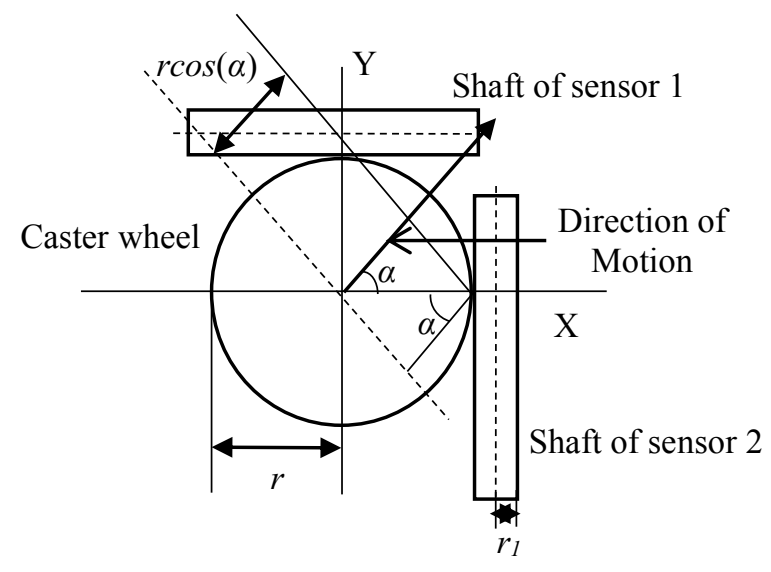

Fig. 1. Assembly of caster wheel along with two sensors

Consider direction of motion of caster wheel with an angle $\alpha$ with respect to positive $\mathrm{x}$ axis as shown in Fig. 1. Shaft 1 and shaft 2 measures $X$ and Y components respectively. Number of rotations the shaft 2 covers if the caster wheel rotates with an angular velocity $\omega$, is given by Eq. (3),

$$
N_{r 2}=\frac{(r \cos (\alpha)) \omega t}{2 \pi r_{1}}
$$


Number of rotations the shaft 1 covers, if the caster wheel rotates with an angular velocity $\omega$ is given by Eq. (4),

$$
N_{r l}=\frac{(r \sin (\alpha)) \omega t}{2 \pi r_{1}}
$$

Sensor readings (X, Y) can be obtained from Eq. (5) and Eq. (6). Here, $\alpha$ is the heading angle, $\omega$ is the angular velocity of caster wheel and $N_{s}$ is the resolution of sensor. The errors occur due to nature of surface over which caster wheel operates, slipping of wheels and effect of contamination on its surface were considered in the model. The errors were assumed to have Gaussian characteristics. Total effect of these errors was incorporated as random fluctuations that occur to the radius of the castor wheel. Maximum value of this error was assumed to be $10 \%$ of the actual dimension of the castor wheel. It was assumed that other errors such as misalignment in the axes of sensor shafts and caster wheel is constant and will be compensated during calibration.

$$
\begin{aligned}
& X=\frac{((10 \cdot r+\operatorname{rand}(-11) \cdot r) \cos (\alpha)) \omega t N_{s}}{20 \pi r_{1}} \\
& Y=\frac{((10 \cdot r+\operatorname{rand}(-1,1) \cdot r) \sin (\alpha)) \omega t N_{s}}{20 \pi r_{1}}
\end{aligned}
$$

Considering inverse kinematics, angular velocity $(\omega)$ of caster wheel and heading angle $(\alpha)$ can be estimated from outputs of two sensors. Values of for the same are calculated by solving the two simultaneous Eq. (5) and Eq. (6). On dividing Eq. (6) by Eq. (5), Eq. (7) is obtained.

$$
\frac{Y}{X}=\frac{(r \sin (\alpha)) \omega t N_{s}}{2 \pi r_{I}} \times \frac{2 \pi r_{I}}{(r \cos (\alpha)) \omega t N_{s}}
$$

Heading angle $(\alpha)$ is given by Eq. (8),

Using trigonometric equation,

$$
\alpha=\tan ^{-1}\left(\frac{Y}{X}\right)
$$

$$
\sin ^{2} \alpha+\cos ^{2} \alpha=1
$$

Eq. (5) and Eq. (6) are altered to get Eq. (10),

$$
Y^{2} \times\left(\frac{2 \pi r_{1}}{r \omega t N_{s}}\right)^{2}+X^{2} \times\left(\frac{2 \pi r_{1}}{r \omega t N_{s}}\right)^{2}=1
$$

From Eq. (10), Angular velocity ( $\omega$ ) of caster wheel can be obtained as given in Eq. (11),

$$
\omega=\frac{2 \pi r_{1}}{r \omega t N_{s}} \times \sqrt{X^{2}+Y^{2}}
$$

\section{Results and discussion}

The sensor output model developed in the previous section was simulated with the help of MATLAB version 8.6. Initial simulations were carried out to analyse output of sensors when the castor wheel was moving in predefined patterns. The effectiveness of this method 
was found out by estimating path tracked by robot with the help of sensor output. Simulations were conducted by considering diameter of caster wheel as $15 \mathrm{~mm}$, diameter of shafts as $1 \mathrm{~mm}$ and resolution of sensor as 30 .

\subsection{Continuously varying heading angle}

Simulations were conducted by continuously varying heading angle of castor wheel from 0 to $2 \pi$. The angular velocity of caster wheel was fixed at $4 \mathrm{rad} / \mathrm{sec}$. The output of sensors during simulation run was calculated and plotted. Fig. 2. (i) and (ii) shows readings of sensor 1 and sensor 2 with respect to variation in heading angle for uniform angular velocity of caster wheel. It was observed from the graphs that the output of both sensors were varying sinusoidally. Output of sensor 2 was leading sensor 1 output by a phase angle of $\pi / 2$. This is due to positioning of sensor 2 perpendicular to $\mathrm{x}$ axis of wheel. Since the wheel rolls towards $\mathrm{x}$ direction initially, maximum readings is obtained at sensor 2 and minimum at sensor 1 . The readings will vary sinusoidally as direction of wheel motion varies. Thus the sensor output was varying as predicted.
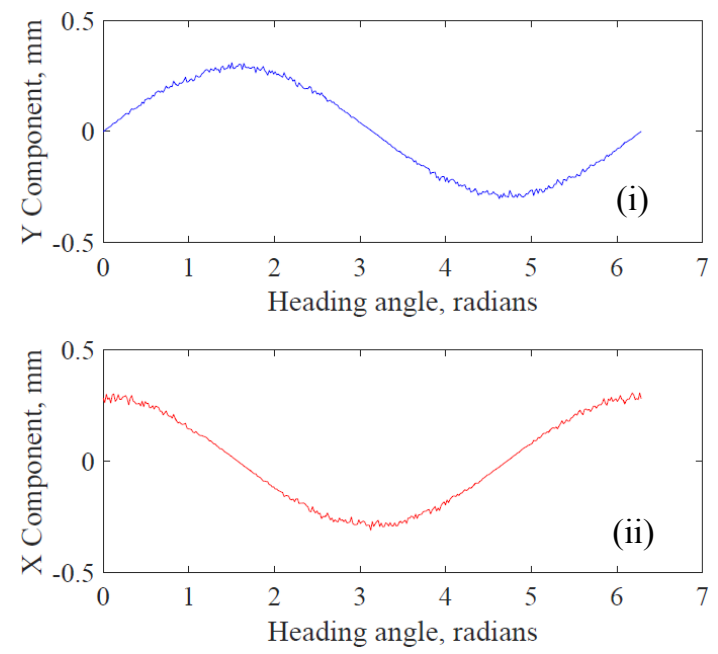

Fig. 2. Sensor output for varying heading angle (i) sensor 1 (ii) sensor 2

\subsection{Continuously varying heading angle and angular velocity of caster wheel}

In order to study variation pattern of sensor readings when both input parameters are varied periodically, another simulation was carried out. Values of heading angle and angular velocity of caster wheel was taken as $\alpha=\sin (2 t)$ and $\omega=\sin (t)$. Fig. 3. depicts variations in (i) heading angle and (ii) angular velocity of caster wheel with respect to time. Based on the obtained periodic values of heading angle and angular velocity of caster wheel, sensor output was calculated. The plot of sensor output when heading angle and angular velocity of caster wheel are considered to be time dependent is as shown in Fig. 4. (i) and (ii). It can be clearly seen that sensor output readings vary proportionally as the time dependent input parameters fluctuates. Readings of sensor 1 and sensor 2 complement each other since the direction of wheel motion determines the output of sensors. Similar to the above case, it can be seen that the senor readings vary accordingly as the direction of wheel changes. 

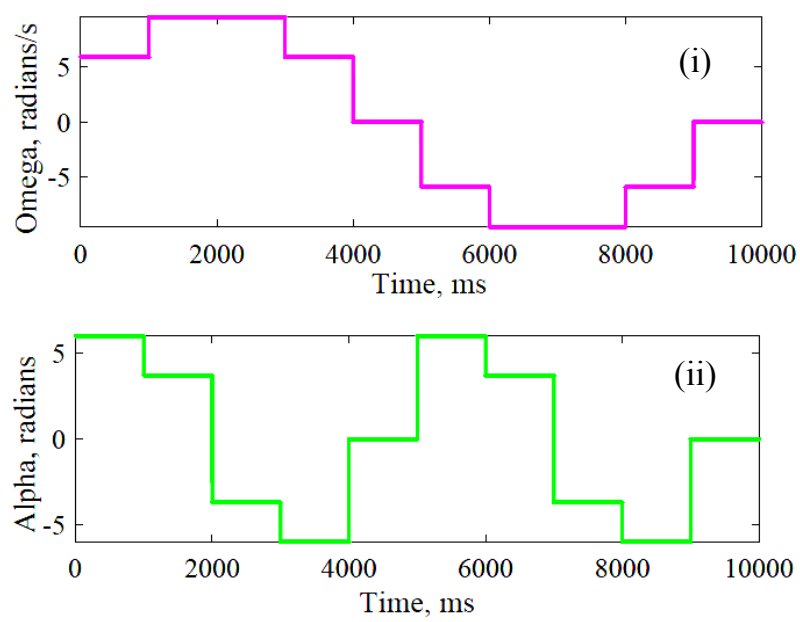

Fig. 3. Variations in (i) angular velocity, $\omega($ ii) heading angle, $\alpha$
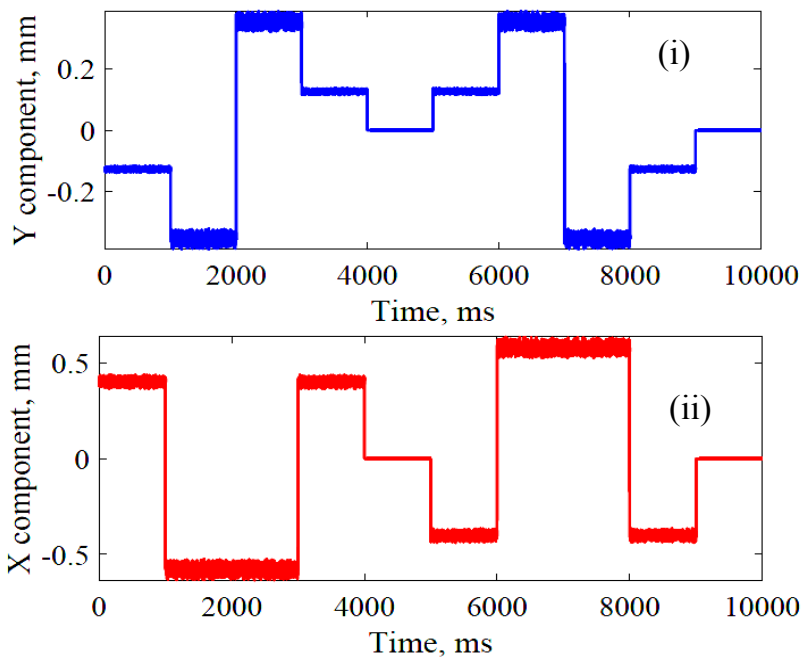

Fig. 4. Output responses when $\omega$ and $\alpha$ are time dependent quantities (i) sensor 1 (ii) sensor 2

\subsection{Motion along a regular geometrical trajectory}

Path traced by a mobile robot can be predicted with the help of sensor readings. For simplicity, consider path traced by mobile robot to be a rectangle of $150 \mathrm{~mm}$ by $75 \mathrm{~mm}$ as shown in Fig. 5. In this regard the heading angle and angular velocity of caster wheel was kept as given in Fig. 6 (i) and (ii). With input parameters as mentioned earlier, output of both sensors is obtained which is illustrated in Fig. 7 (i) and (ii). It is evident from the Fig. 5 that initially the output was only obtained from sensor 2 . This is because initially heading angle was at $0 \mathrm{rad}$, which indicates that the robot motion was towards $\mathrm{x}$ direction. Similar to above discussed cases, the sensor readings vary according to the change in direction of wheel motion.

Concept of inverse kinematics was implemented to verify the correctness of sensor readings obtained. In addition to prove that the actual path tracked by robot can be retrieved 
from sensor readings. According to the concept, angular velocity of caster wheel and heading angle were estimated from sensor readings using established Eq. (5) and Eq. (6).

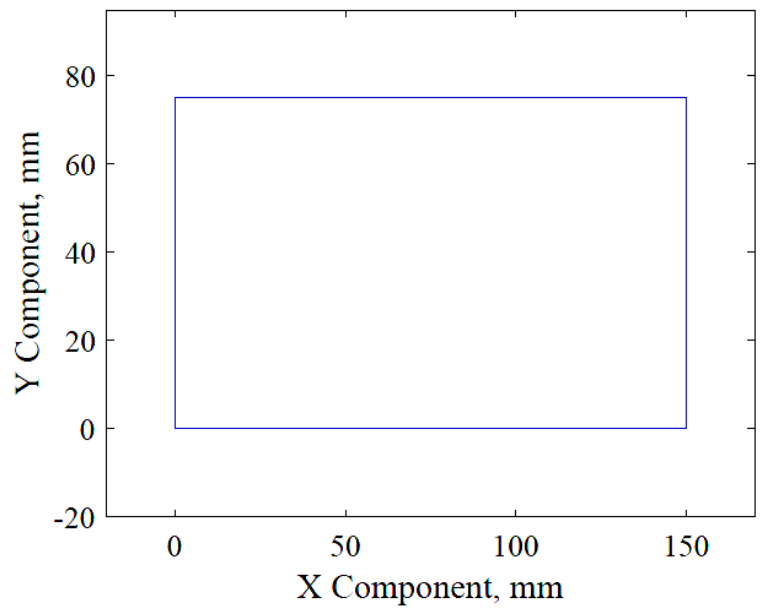

Fig. 5. Path traced by the mobile robot
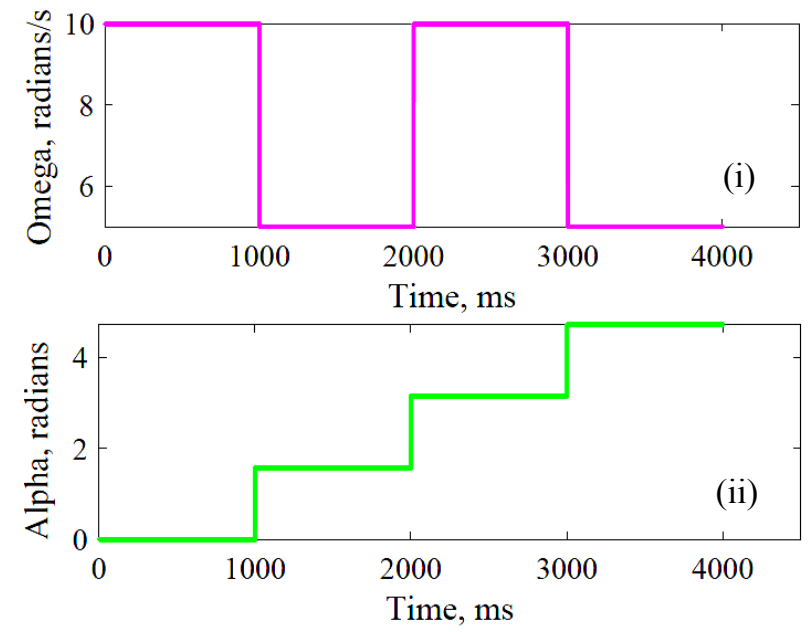

Fig. 6. Input parameters (i) angular velocity, $\omega$ (ii) heading angle, $\alpha$

A comparison was made between actual and estimated values of input parameters $(\omega$ and $\alpha$ ) for further analysis. Fig. 8 (i) and Fig. 8 (ii) depicts the plot of actual and estimated values of angular velocity of caster wheel and heading angle with respect to time respectively. From Fig. 8 (ii), it can be observed that the estimated value of angular velocity is affected by the disturbances and sources of error. The trend with which actual and estimated angular velocity varies is similar.

Similarly, in Fig. 8 (i), the estimated values of heading angle are in accordance with that of the actual values. The deviation in estimated heading angle from actual heading angle after $3 \mathrm{~s}$ is due to the inherent limitation of $\tan ^{-1}$ trigonometric function in inverse kinematic equations to give value greater than $\pi$ (i.e., $\pi+x=-\pi+x$ ). Thus this error in predicted heading angle will not contribute to any other errors. Therefore, it can be stated that the algorithm deduced in the previous section holds good for the purpose of position estimation of mobile robot. 

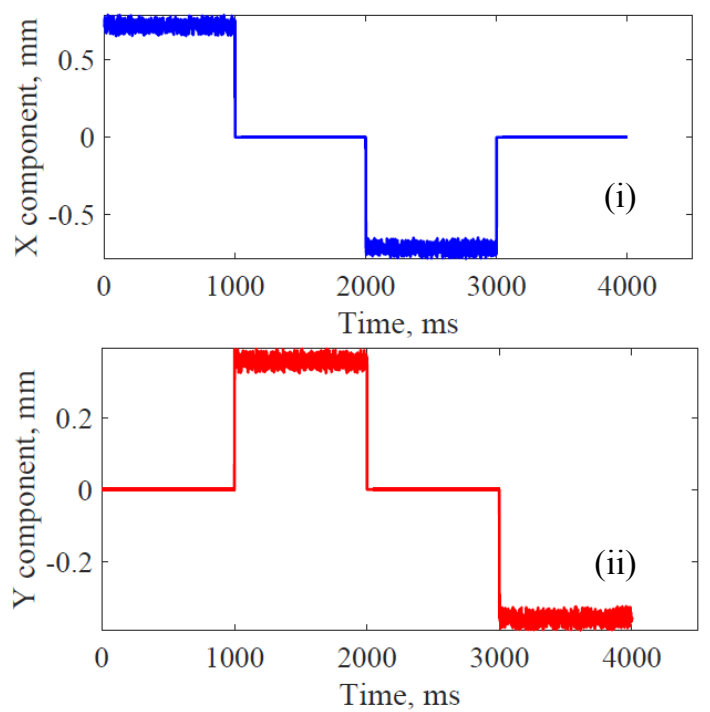

Fig. 7. Sensor readings (i) Sensor1 (ii) Sensor 2
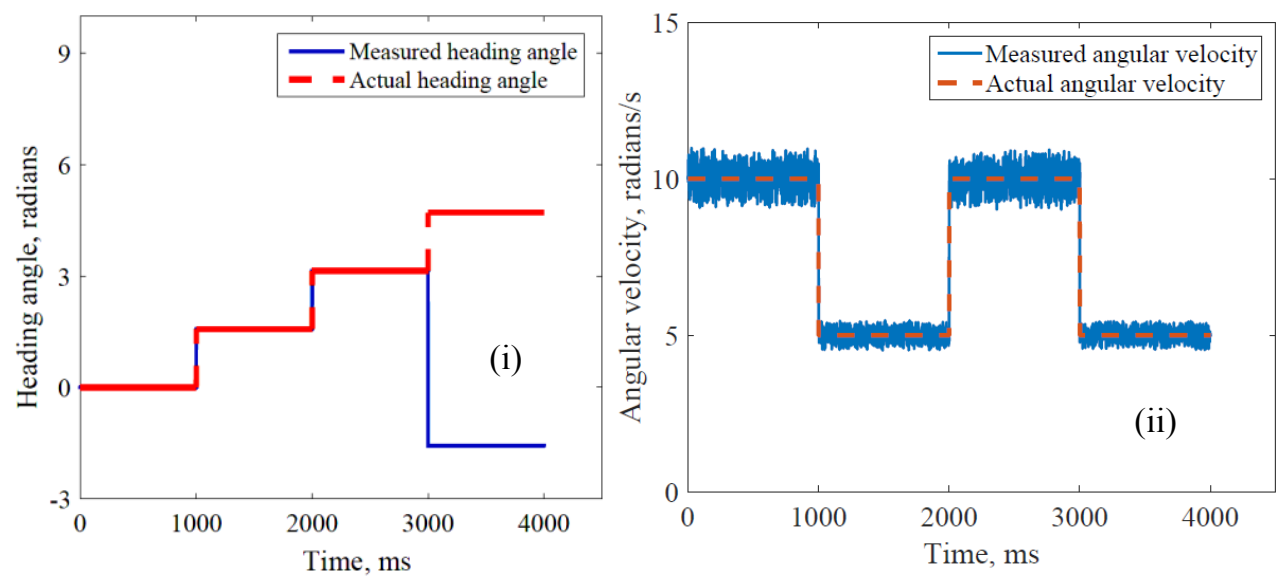

Fig. 8. Actual and estimated values (i) angular velocity of caster wheel (ii) heading angle

The verification is further continued by predicting path traced by mobile robot using estimated values of angular velocity of caster wheel and heading angle and established Eq. (5) and Eq. (6). This was conducted to analyse the path traced by mobile robot when estimated values of input parameters were considered. Fig. 9 makes a comparison between the path traced by mobile robot while considering the actual and estimated values of input parameters. It is worth to notice that the path tracked using actual and estimated values of input parameters are in good agreement. The effect of sensor errors is minimal on the estimated trajectory. This justifies that the position estimation of mobile robot by sensing caster wheel motion is possible with an appropriate hardware set-up. 


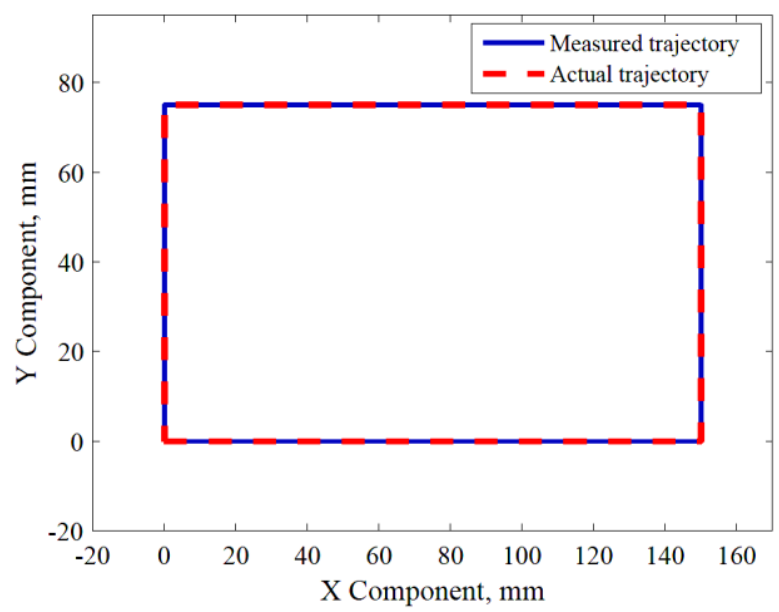

Fig. 9. Comparison between the path traced by the mobile robot while considering the actual and estimated values

\section{Conclusions}

The paper presented a mathematical model developed to estimate the position of mobile robot by sensing the motion of caster wheel. Different sources of errors that will occur during sensing were included in the model. A pilot simulation study was carried out to establish the effectiveness of method in detection of motion and position estimation. Sensor reading was calculated in different scenarios of castor wheel motion with different input parameters. The sensor readings are further used to recalculate the input parameters and compared with the actual input parameters. The sensor output when the robot was tracking a rectangular trajectory of $150 \mathrm{~mm} \times 75 \mathrm{~mm}$ was also simulated. The robot was assumed to run at $150 \mathrm{~mm} / \mathrm{s}$ in lengthy sides of the rectangular trajectory and $75 \mathrm{~mm} / \mathrm{s}$ at short sides. Total travel time of 4 second was obtained. The results obtained from simulation show that the calculated and the actual values of input parameters, viz angular velocity of caster wheel and heading angle are on par. It is also observed from the simulation results that, the path traced by mobile robot can be determined from the output of the sensors although the sensors are exposed to external noises. On considering the results, it is seen that the concept can be experimented on a real mobile robot for estimation of its position.

\section{References}

1. M. Jamshidi, Robotics and Remote Systems for Hazardous Environments Prentice Hall, (1993)

2. S.G. Tzafestas, Introduction to Mobile Robot Control, Elsevier, (2014)

3. M. Endo, K. Hirose, Y. Hirata, Inter. Conf. on Intelligent Robots and Systems, 27952801, (2008)

4. J.M. Usher, R. McCool, L. Strawderman, Simulation Modelling Practice and Theory, 75, 96-112, (2017)

5. N. Chivarov, D. Chikurtev, K. Yovchev, IFAC-Papers On Line, 48, 24, 128-133, (2015) 
6. N. Yonezawa, K. Kosuge, Y. Hirata, Proc. of the 2012 IEEE Int. Conf. on Robotics and Biomimetics, 1874-1880, (2012)

7. M. Endo, K. Hirose, Y. Hirata, Proc. of the 2009 IEEE Int. Conf. on Robotics and Automation, 2350-2355, (2009)

8. A. Lottermoser, C. Berger, S. Braunreuther, Procedia CIRP, 62, 594-599, (2017).

9. N. Ruangpayoongsak, H. Roth, J. Chudoba, Proc. of the 2005 IEEE Int. Workshop on Safety, Security and Rescue Robotics, 212-217, (2005)

10. C. Suliman, C. Cruceru, F. Moldoveanu, Scientific Bulletin of the Petru Maior University of TirguMures, (2009)

11. M. Cimino, P.R. Pagilla, Automatica, 47, 10, 2267-2272, (2011)

12. W. Burgard, A. Derr, D. Fox, A.B. Cremers, Proc. of the 1998 IEEE Int. Conf. on Intelligent Robots and Systems, 2, 730-735, (1998).

13. S. Se, D. Lowe, J, Little, Proc. of the 2001 IEEE/RSJ Int. Conf. on Intelligent Robots and Systems, 1, 414-420, (2001)

14. D. Fox, W. Burgard, S. Thrunz, Proc. of the Fifteenth National Conf. on Artificial Intelligence, 983-988, (1998) 